\title{
Localisation et estimation des volumes de déchets suite à l'ouragan Irma à Saint-Martin
}

\author{
Location and estimation of waste volumes following Hurricane Irma in
} Saint-Martin

\author{
Anne Ruas ${ }^{1}$, Serge Lhomme ${ }^{2}$, Michelet Lamour ${ }^{1}$ \\ ${ }^{1}$ Université Gustave Eiffel, Laboratoire COSYS/LISIS, F-77447 Marne la Vallée, France \\ ${ }^{2}$ Univ Paris Est Creteil, LAB'URBA, F-94010 Creteil, France
}

\begin{abstract}
RÉSUMÉ. Les ouragans génèrent de grandes quantités de déchets directement liés aux impacts induits. La gestion de ces déchets constitue alors un enjeu majeur de la gestion de crise post-ouragan. L'ouragan Irma qui a touché les Caraïbes début septembre 2017, en particulier les îles de Saint-Martin et de Saint-Barthélemy, est un exemple frappant de cette problématique. Ce travail s'insère dans le projet ANR DéPOs qui vise à proposer des outils permettant d'améliorer la collecte des déchets post-ouragans dans les Antilles françaises. Dans ce cadre, nous présentons des analyses relatives à la localisation des bâtiments détruits et une estimation de la quantité de déchets provenant des bâtiments. Les recherches menées ont abouti à la conception d'un modèle d'analyse de la collecte des déchets post-ouragans et d'une fonction d'estimation des volumes de déchets générés par un ouragan lorsque les impacts sont connus. Ces recherches constituent une première étape dans le développement d'un système d'information devant permettre d'améliorer la collecte des déchets post-ouragan dans les Antilles françaises.

MOTS-CLÉS. Déchets post catastrophe, Ouragan, Caraïbes, Gisement, Gestion des déchets.
\end{abstract}

\section{L'ouragan Irma à Saint Martin et l'enjeu d'estimation des volumes de déchets}

L'ouragan Irma a touché les Caraïbes au début de septembre 2017. L'ouragan Irma est classé en catégorie 5 sur l'échelle de Saffir-Simpson et fait partie des ouragans les plus violents de l'Atlantique Nord, avec des vents extrêmement forts $(287 \mathrm{~km} / \mathrm{h})$ et des rafales pouvant atteindre $320 \mathrm{~km} / \mathrm{h}$ (Cangialosi et al., 2018), voire $370 \mathrm{~km} / \mathrm{h}$ selon les sources (Gustin, 2017). Sur sa trajectoire, les îles de Saint-Martin et de Saint-Barthélemy ont été particulièrement touchées. Les dommages ont été causés par la violence du vent, mais également par la montée des eaux qui accompagne les ouragans. Une carte de submersions marines suite à IRMA réalisée par le CEREMA, identifie des surcotes de 2 à 3 mètres au-dessus du niveau de la mer, dans certains quartiers (Sandy Ground et d'Orléans, figure 8). Ainsi des véhicules, des toits de maison ont été emportés par le vent, des vitres brisées et l'eau de mer, salée, s'est infiltrée dans les habitations. Les habitations qui ont perdu toit et vitres ont également subi des dégradations de mobiliers et d'appareils électriques dues aux pluies.

Selon les données du programme spatial européen Copernicus, 19,7 \% des bâtiments à SaintMartin et 2,5 \% à Saint-Barthélemy ont été totalement détruits ou sévèrement endommagés. Le coût total des dommages assurés est estimé à près de 2 milliards d'euros par les compagnies d'assurance : 1,17 milliard pour Saint-Martin et 823 millions pour Saint-Barthélemy (https://www.gouvernement.fr/etat-des-lieux-depuis-irma).

Les déchets générés par ces impacts représentent alors un enjeu majeur dans la gestion de crise post-ouragans. Dans un premier temps, les déchets dispersés sur le territoire gênent la progression des services de secours et des services techniques de l'assainissement, de la production et de la distribution d'eau, d'énergie et de communication (Beraud et al., 2019 ; Vinet et al., 2019). Dans un deuxième temps, les quantités hors normes de déchets désorganisent les filières de traitement habituelles et leur mauvaise gestion peut provoquer des dommages sanitaires, environnementaux et 
psychologiques à court ou moyen terme (prolifération de moustiques et autres nuisibles, brûlages à l'air libre, pollutions des sols, de l'air, des eaux douces et marines, etc.), des inégalités sociales (rythme et choix de zones à déblayer) et une dégradation de l'image et de l'attractivité des territoires (Beraud et al., 2019 ; Vinet et al., 2019). Ces problématiques sont présentes pour d'autres aléas comme les inondations ou les tremblements de terre, c'est pourquoi la littérature sur la gestion des déchets post-catastrophe est aujourd'hui conséquente (Lauritzen, 1998 ; Mc Entire, 2006 ; Brown et al., 2011 ; Béraud, 2013 ; Tabata et al., 2019 ; Zhang et al., 2019).

Le travail présenté s'inscrit dans le cadre du projet DéPOs, financé par l'Agence Nationale de la Recherche, ANR-18-OURA-0003. Si le projet cherche à améliorer la collecte des déchets postouragans dans les Antilles françaises, dans cet article sont présentés les analyses relatives à la localisation des bâtiments détruits ou endommagés ainsi qu'à l'estimation de la quantité de déchets provenant des bâtiments sur l'île de Saint-Martin suite à Irma. En effet, une bonne connaissance de la localisation des gisements de déchets est nécessaire pour mieux comprendre comment s'est déroulée la collecte des déchets post-Irma. De plus, travailler sur des méthodes pouvant contribuer à estimer ces gisements avant qu'un ouragan ne se produise sur un territoire (ou très peu de temps après, dès que les premiers impacts sont connus) constitue un enjeu important pour préparer la collecte de ces déchets (USEPA 2008). «Development of a standard method of reporting disaster waste composition and quantities would enable future analysis between events and improve our ability to develop better waste estimation methodologies » (Brown et al., 2011).

Ainsi, grâce à la connaissance des bâtiments détruits et endommagés sur Saint-Martin et aux informations disponibles sur les déchets acheminés à l'unique décharge de l'île, nous essayons de calculer les volumes de déchets en fonction des endommagements et de proposer une modélisation de l'information numérique qui sera nécessaire pour la réalisation de l'algorithme de simulation de la collecte en intégrant les connaissances issues de la composante organisationnelle de la collecte (Beraud et al, 2019). Il existe déjà de nombreuses méthodes cherchant à quantifier les déchets générés par une catastrophe (Chen et al., 2007 ; Hirayama, 2010 ; FEMA, 2018), des revues de la littérature existent même pour tenter de les répertorier et de les classer (Marchesini et al., 2021). Néanmoins, compte tenu des données et des outils disponibles, des spécificités des territoires et des aléas étudiés, il est dans les faits très souvent difficile de trouver des méthodes existantes parfaitement adaptées au contexte d'analyse. Les résultats obtenus avec la méthode proposée ici seront donc comparés à une seule méthode : la méthode California (Office of Emergency Service OES CALIFORNIA, 2005). Cette méthode présente la particularité d'être très utilisée puisqu'elle est relativement simple à mettre en œuvre et semble s'adapter à de nombreux contextes. A noter également que la recherche présentée dans cet article se focalise précisément sur les déchets provenant des bâtiments détruits et endommagés, faute d'informations localisées sur les autres types de déchets, tels que les déchets verts ou les véhicules.

Nous commençons par décrire les données puis la modélisation que nous proposons pour réaliser notre analyse. Cette modélisation sera utilisée pour la conception de l'algorithme de simulation de la collecte des déchets. Une méthode d'estimation des volumes de déchets est ensuite présentée. Les résultats obtenus à l'aide de cette méthode sont comparés aux informations récoltées sur le terrain. Enfin, les analyses spatiales de la localisation des bâtiments détruits et endommagés sur l'île de Saint-Martin suite à l'ouragan Irma sont examinées.

\section{Les données géographiques mobilisées pour l'analyse}

L'enjeu de la recherche est de comprendre l'acheminement des déchets post-catastrophe vers les lieux de traitement et de stockage des déchets et de développer un algorithme de simulation de la collecte. Nous avons donc besoin d'informations géographiques pour comprendre la situation à Saint-Martin, puis pour mettre au point des méthodes numériques d'analyse et de simulation. Ces 
méthodes pourront ensuite être déployées sur d'autres territoires. Autant pour le diagnostic que pour la simulation, nous utilisons des données géographiques numériques. En particulier, nous avons besoin :

- de la localisation des gisements de déchets, c'est-à-dire des bâtiments détruits ou endommagés,

- d'informations sur les bâtiments pour essayer de calculer le volume de déchets provenant des bâtiments endommagés,

- des lieux de stockage de déchets lorsqu'ils sont connus pour comprendre comment transitent les déchets,

- du réseau routier qui est utilisé pour acheminer les déchets de leur origine jusqu'à leur destination,

- d'éléments géographiques contextuels permettant d'étudier la répartition des déchets telles que la distance à la mer, la répartition des habitations ou encore le relief.

Pour les données topographiques, l'île de Saint Martin est couverte par la BDtopo de l'IGN et par des données OSM (OpenStreetMap). Nous avons choisi d'utiliser sur la partie Française de SaintMartin les données de l'IGN parce qu'elles disposent d'information volumétrique sur les bâtiments et altimétrique sur les routes et d'un modèle numérique de terrain. Pour couvrir la partie hollandaise, nous avons utilisé les données OSM de l'île. Les données en raccord entre les parties française et hollandaise de l'île ont été appariées manuellement compte tenu de la petite taille de l'île (Figure 1). Ces données, une fois appariées, nous ont permis de réaliser de nombreuses cartes, dont celles présentées dans ce papier, en fonction des besoins du projet et des analyses.

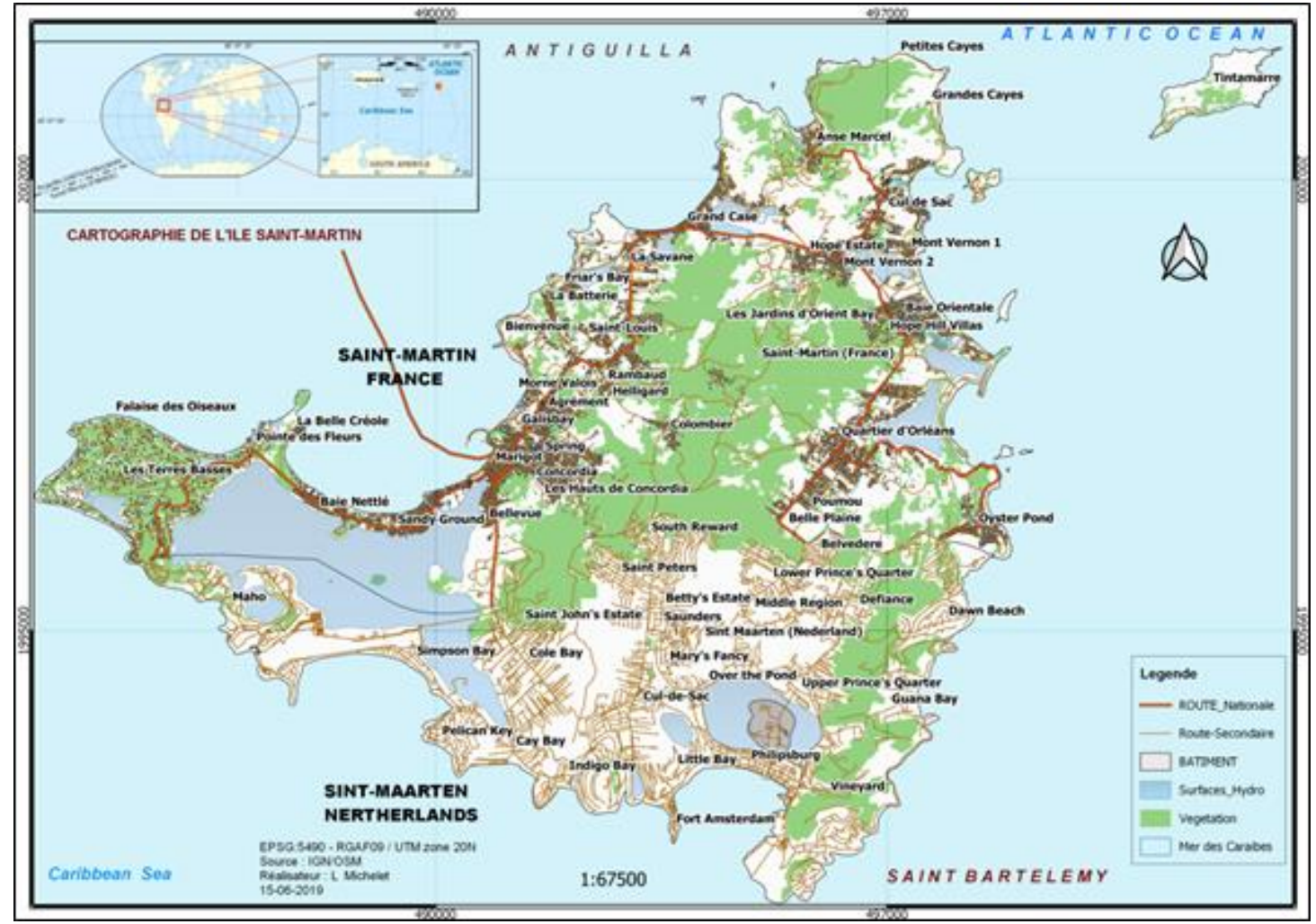

Figure 1. Données de référence sur Saint-Martin. Intégration de données IGN et OSM.

Pour les informations liées aux déchets et à leur collecte, nous avons utilisé trois sources d'informations : une base de données des bâtiments endommagés réalisées par le SERTIT pour le CNES; des données du programme spatial européen COPERNICUS décrivant également les endommagements des bâtiments ; des informations provenant d'enquêtes terrain faites dans le cadre du projet ANR DéPOs pour localiser les zones de dépôt temporaire des déchets. Pour les calculs de volume et de répartition des déchets, les données d'endommagements du SERTIT ont été 
privilégiées, car elles ont été obtenues plus rapidement. Les données d'endommagements COPERNICUS ont été utilisées pour comparer certains résultats.

Les données du SERTIT sont des données ponctuelles qui décrivent, pour chaque bâtiment, quatre niveaux d'endommagement selon la norme EMS-98, en regroupant les classes pas ou peu endommagé. Les bâtiments sont ainsi classés en quatre catégories : "Détruit »; "Fortement endommagé »; «Moyennement endommagé »; «Pas ou Peu endommagé ». Pour associer ces données aux bâtiments de la BDTopo, il suffit en théorie d'appliquer une jointure spatiale puisque ces points correspondent aux centres des bâtiments identifiés par le SERTIT. Néanmoins, les données ponctuelles ne se superposant pas toujours parfaitement aux bâtiments de la BDTopo, un recalage manuel a parfois été nécessaire (Figure 2).
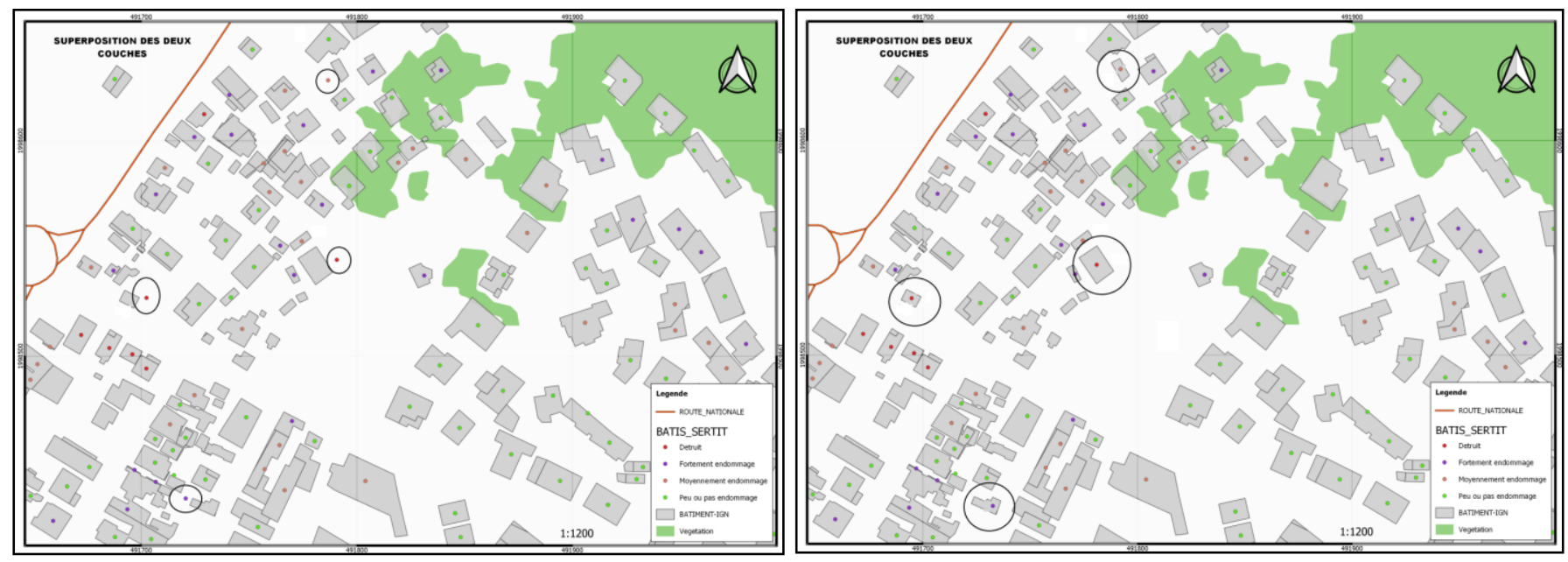

Figure 2. Décalage et recalage des données SERTIT par rapport à la BDTopo dans le quartier de Marigot.

Ainsi, sur les 17090 objets de type bâtis présents dans la BDTopo, 9587 sont appariés avec des informations de la base SERTIT. Une analyse des bâtiments manquants montre qu'il s'agit essentiellement de petits bâtiments ou de petites parties de bâtiments. L'objectif du travail étant d'analyser globalement la répartition spatiale des bâtiments endommagés et des déchets résultants, une information parfaitement exhaustive n'est pas nécessaire. Ainsi, les analyses des répartitions spatiales ont été produites sur ces 9587 objets.

Néanmoins, en ce qui concerne le calcul du volume de déchets, ces problèmes d'appariements sont plus critiques et il convient de mieux comprendre la nature de ces 7503 polygones oubliés. C'est pourquoi une analyse statistique fine s'est révélée nécessaire. Les objets manquants sont des objets de petites tailles $\left(37 \mathrm{~m}^{2}\right.$ en moyenne) et cumulent une surface représentant $10 \%$ de la surface globale. Ces objets manquants sont soit des petits bâtiments qui n'ont pas été classés du fait de leur petite taille, soit des extensions de bâtiments dont l'omission vient du découpage des données, soit des oublis de saisie.

Une analyse plus fine montre en effet que 6545 des 7503 polygones (bâtiments) sont situés à moins de 5 mètres des bâtiments appariés avec les données d'endommagement du SERTIT, indiquant ainsi que des imprécisions sont a priori à l'origine de ce nombre relativement important d'objets manquants. Ces polygones ont une taille moyenne de $32 \mathrm{~m}^{2}$ ce qui correspond bien à des découpages différentiés de la BDTopo, souvent en raison de hauteurs de bâtiments différentes, mais non différentiés dans les données SERTIT. Il ne s'agit pas dans ce cas de nouveaux objets mais de surfaces que nous intégrons dans la synthèse lorsqu'elles sont proches de maisons détruites ou endommagées (Tableau 1). Les autres objets qui semblent être oubliés des données du SERTIT sont de plus grande taille. Nous faisons l'hypothèse dans ce cas qu'il s'agit d'objets «pas ou peu 
endommagés » qui n'ont pas été saisis. Par conséquent, leur absence ne nuit pas au calcul des volumes de déchets.

\begin{tabular}{|c|c|c|c|c|c|}
\hline $\begin{array}{c}\text { Niveau d' } \\
\text { endommagement } \\
\text { des bâtiments }\end{array}$ & $\begin{array}{c}\text { Nombre de } \\
\text { bâtiments } \\
\text { appariés } \\
\text { (superposés aux } \\
\text { données SERTIT) }\end{array}$ & $\begin{array}{c}\text { Pourcentage des } \\
\text { bâtiments } \\
\text { appariés } \\
(9587 \\
\text { bâtiments) }\end{array}$ & $\begin{array}{c}\text { Surface } \\
\text { totale des } \\
\text { bâtiments } \\
\left(\mathrm{m}^{2}\right)\end{array}$ & $\begin{array}{c}\text { Ajout des } \\
\text { surfaces des } \\
\text { bâtiments } \\
\text { proches }\end{array}$ & $\begin{array}{c}\text { Somme totale } \\
\text { des surfaces } \\
\text { corrigées }\left(\mathrm{m}^{2}\right)\end{array}$ \\
\hline Détruits & 659 & $6,9 \%$ & $\mathbf{7 7 6 0 6}$ & 12796 & $\mathbf{9 0 4 0 2}$ \\
\hline $\begin{array}{c}\text { Fortement } \\
\text { endommagés }\end{array}$ & 1624 & $16,9 \%$ & $\mathbf{3 0 0 9 1 8}$ & 43980 & $\mathbf{3 4 4 8 9 8}$ \\
\hline
\end{tabular}

Tableau 1. Comptage des bâtiments détruits et fortement endommagés sur Saint-Martin à partir des donnés SERTIT.

Nous comparons enfin ces données avec les données COPERNICUS que nous avons pu acquérir après nos premières analyses. Pour rappel, COPERNICUS est un programme d'observation porté par l'Europe qui permet le suivi des risques naturels sur l'Europe et dans le monde en utilisant des images spatiales dans le cadre de son programme EMS (Emergency Management System). Les données COPERNICUS sur IRMA sont issues du programme EMSN-049, commandé par la Direction Générale de la Sécurité Civile et de la Gestion de Crise. Les données ont été saisies par une entreprise grecque (GEOAPIKONISIS S.A.P.GE) à partir d'un ensemble de sources. Une analyse du processus (EMSN-049, 2018, p23) montre que les données de l'IGN ont amplement été utilisées (dont la BDTopo et l'Ortho HR de 2017) ainsi que des images Pléiades. Comme les données d'endommagement du SERTIT, ces données utilisent la nomenclature EMS-98 pour la classification des bâtiments endommagés, essentiellement par photo-interprétation. Le rapport (EMSN-049, 2018) comptabilise 13163 bâtiments dont 2526 (19,2\%) détruits et 1354 fortement endommagés (10,3\%) (EMSN-049, 2018, p48). Ces données ont été ultérieurement acquises, modifiées et complétés par la DEAL de Guadeloupe. Ainsi les chiffres issus des données d'endommagement du SERTIT et des données COPERNICUS mises à jour par la DEAL sont sensiblement différents (Tableau 2).

\begin{tabular}{|l|c|c|c|c|c|}
\hline $\begin{array}{c}\text { Niveau } \\
\text { d'endommagement des } \\
\text { bâtiments }\end{array}$ & $\begin{array}{c}\text { SERTIT } \\
\text { Nombre de } \\
\text { bâtiments }\end{array}$ & $\begin{array}{c}\text { SERTIT } \\
\text { Surface } \\
\left(\mathrm{m}^{2}\right)\end{array}$ & $\begin{array}{c}\text { SERTIT } \\
\text { Corrigées } \\
\left(\mathrm{m}^{2}\right)\end{array}$ & $\begin{array}{c}\text { COPERNICUS/ } \\
\text { DEAL } \\
\text { Nombre de } \\
\text { bâtiments }\end{array}$ & $\begin{array}{c}\text { COPERNICUS / } \\
\text { DEAL } \\
\text { Surface }\left(\mathrm{m}^{2}\right)\end{array}$ \\
\hline Détruits & $659(6,9 \%)$ & $\begin{array}{c}77606 \\
(2,8 \%)\end{array}$ & $\begin{array}{c}90402 \\
(3,2 \%)\end{array}$ & $\begin{array}{c}2732 \\
(12,8)\end{array}$ & 233096 \\
\hline Fortement endommagés & $1624(16,9 \%)$ & 300918 & 344898 & 1511 & 278649 \\
& & $(10,8)$ & $(12,3 \%)$ & $(7,1 \%)$ & $(9,9)$ \\
\hline Total & 2283 & 378524 & $\mathbf{4 3 5 3 0 0}$ & 4243 & $\mathbf{5 1 1 7 4 5}$ \\
& $(23,8 \%)$ & $(13,5)$ & $(15,6)$ & $(19,9)$ & $(18,2)$ \\
\hline
\end{tabular}

Tableau 2. Comptage des bâtiments détruits et fortement endommagés entre source SERTIT et COPERNICUS 
Une comparaison des données et une analyse de photographies prises sur le terrain dans le cadre du projet DéPOs (Nithart et al, 2018, Perez 2019) montre une assez bonne cohérence entre les classifications, même si, on note des écarts d'interprétation comme illustré en figure 3. Les données COPERNICUS sont un peu plus «pessimiste » que les données SERTIT ce qui se voit également dans le tableau 2.
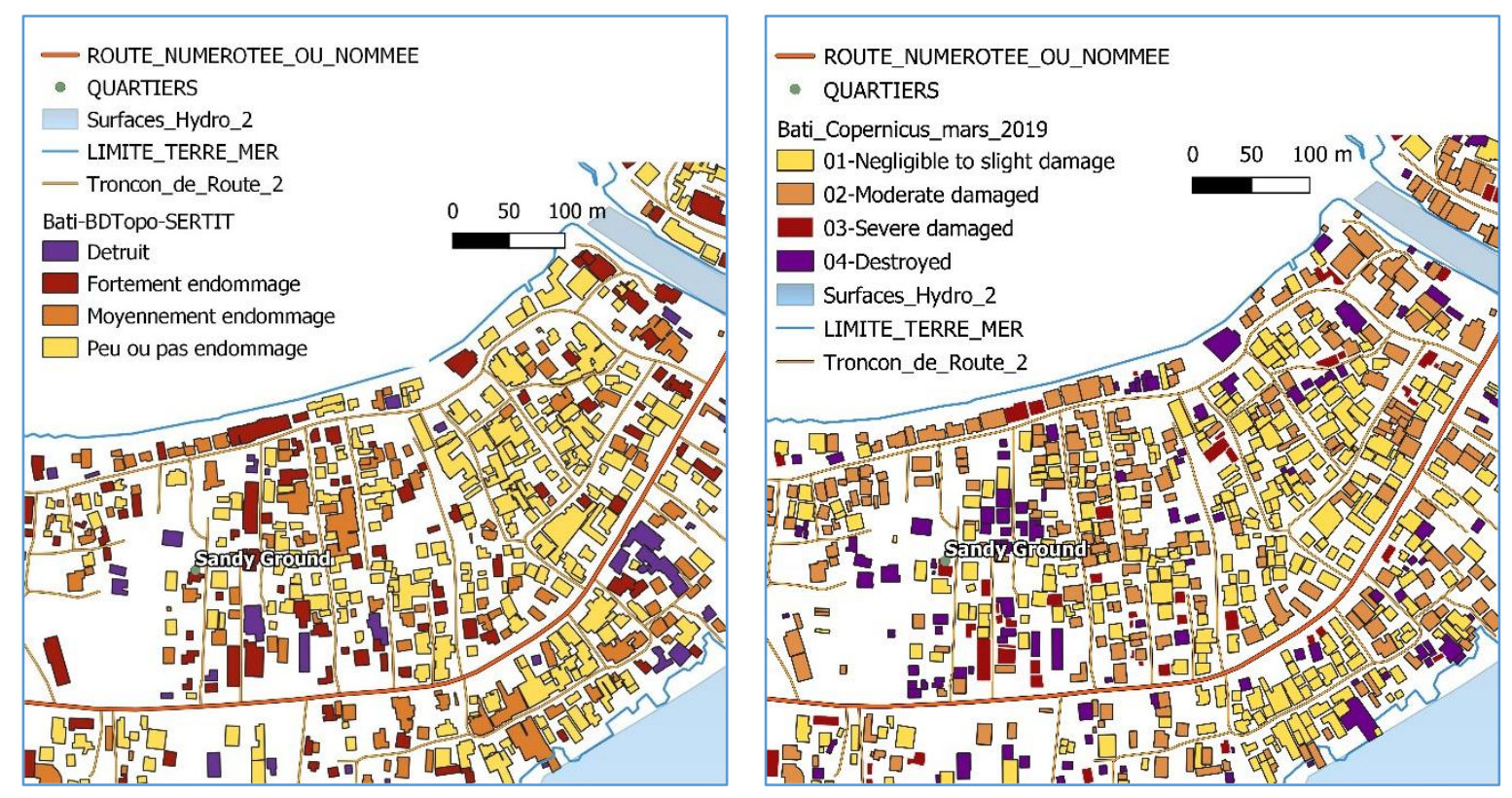

Figure 3. Différence d'interprétation des endommagements selon les sources SERTIT (gauche) ou Copernicus (droite).

Même si ces données d'endommagement sont imparfaites (imprécises et incomplètes), ces informations sont précieuses parce qu'elles nous permettent d'analyser la localisation des gisements de déchets et de concevoir un système de simulation de la collecte pour aider à l'amélioration des choix et pratiques pour la collecte de déchets lors de futurs ouragans.

\section{Modélisation des données relatives aux bâtiments endommagés et aux lieux de collecte des déchets}

Grâce aux différentes missions de terrain faites dans le cadre de projets DéPOs (Nithart et Bonnemains, 2018; Popescu et Perez, 2019; Perez, 2019) il a été possible de comprendre le processus de collecte des déchets et les acteurs impliqués (Beraud et al., 2019). Ces informations nous ont permis de concevoir une modélisation nécessaire à la simulation de la collecte.

De façon synthétique (Figure 4), les habitants apportent les déchets provenant de leur habitation endommagée aux aires de dépose proches de leur logement. Les déchets sont ainsi parfois déposés juste devant les maisons, mais d'autres fois ils sont regroupés sur des aires désignées. Des entreprises, dans un bassin de collecte transportent ces déchets par camion des aires de dépose à des zones de transit (parfois appelées zones de stockage temporaires) désignées par les autorités. Les déchets sont dans un troisième temps apportés des zones de transit à la décharge officielle de type ISDND (Installation de Stockage de Déchets Non Dangereux). Les aires de dépose sont en principe éphémères même si certaines sont encore visibles 9 mois après l'ouragan. Une partie des déchets peut également être évacuée de façon illégale dans des décharges illégales ou être rejetée dans la mer. Une autre partie est brulée ou fait l'objet de trafic. 


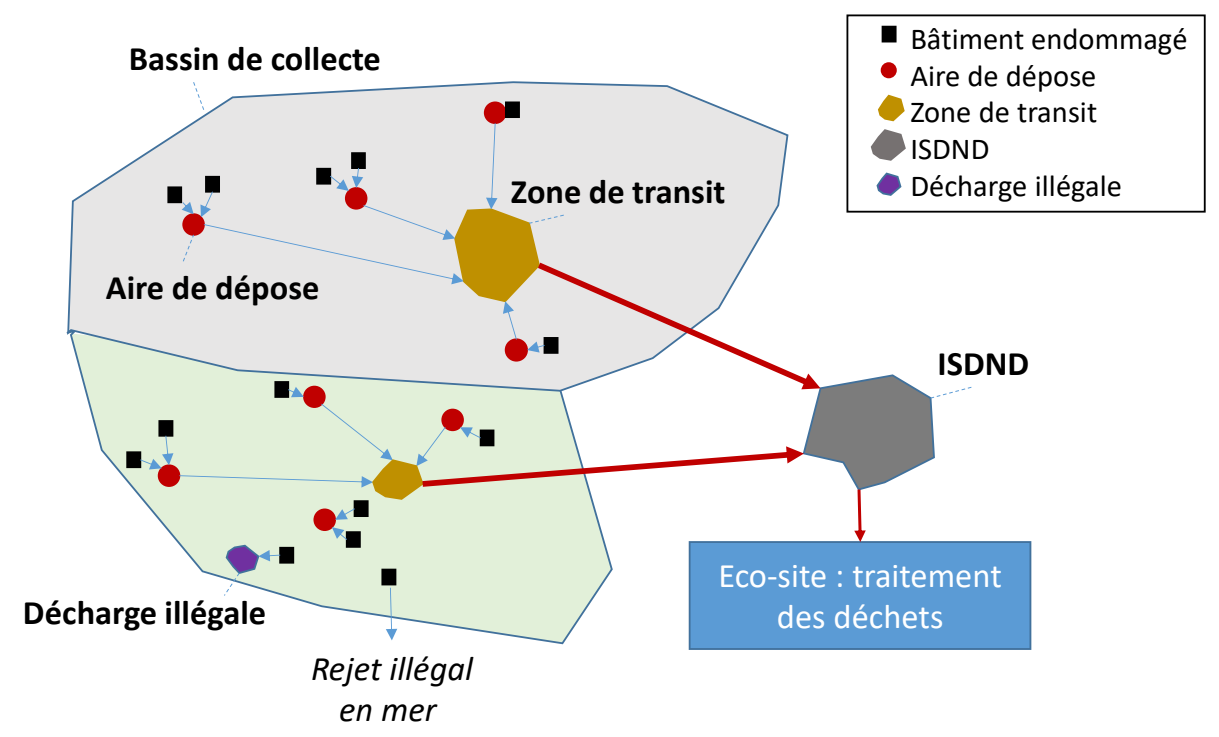

Figure 4. Schéma du déplacement des déchets et sa nomenclature.

Ces informations permettent de construire un schéma de données (Figure 5) utile pour les analyses et les simulations.

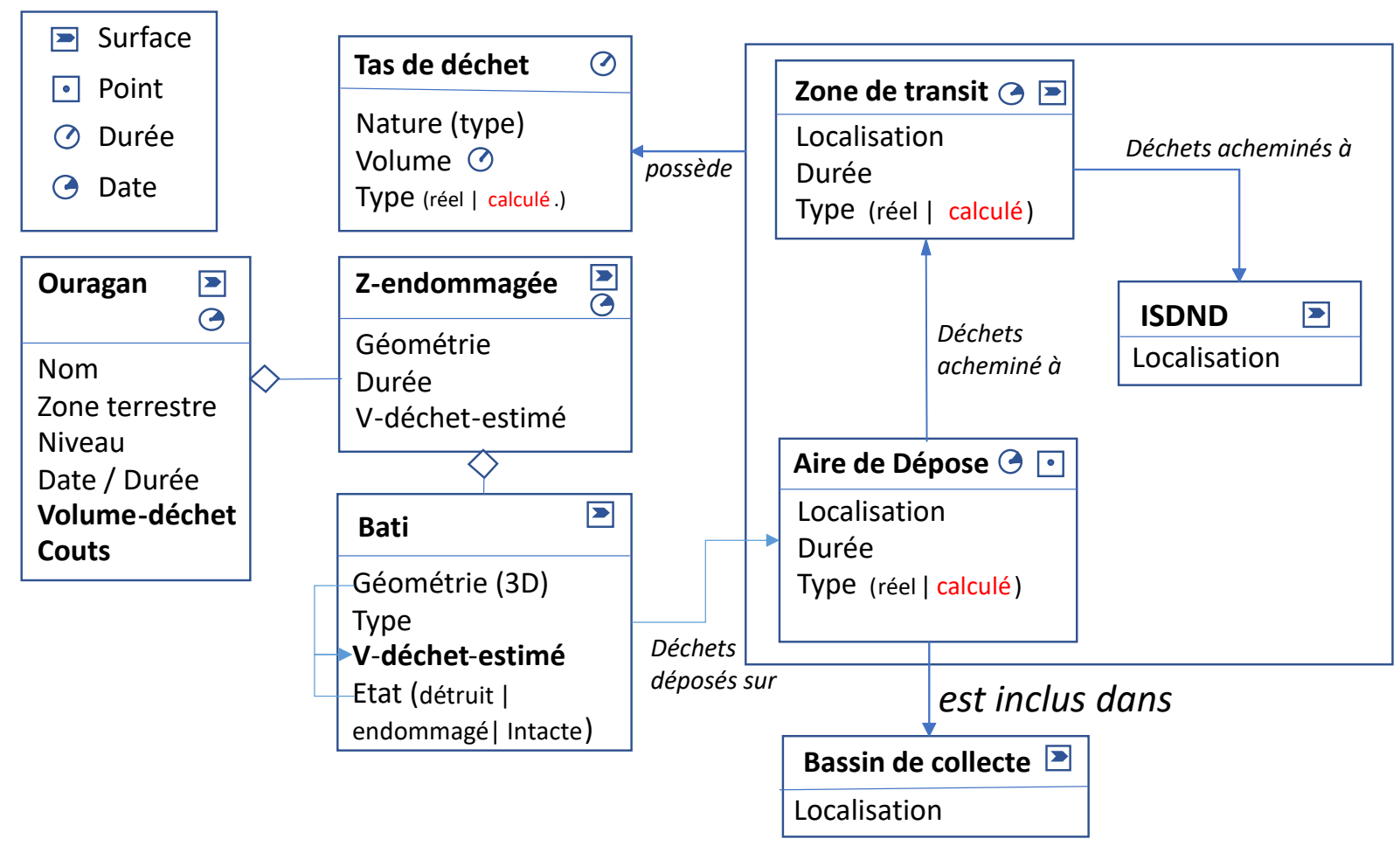

Figure 5. Schéma conceptuel des zones de déchets.

\section{Estimation des volumes de déchets provenant des habitations}

A partir des données d'endommagement présentées en section 2, un des objectifs du projet est d'estimer les volumes de déchets. Pour cela, il existe des méthodes reconnues, comme la méthode California de l'OES CALIFORNIA Débris Management in Disaster Recovery, (Office of Emergency Service OES CALIFORNIA, 2005) qui estime que le volume de débris pour les maisons détruites représente $1 / 3$ du volume des maisons. Néanmoins, cette méthode n'est pas parfaitement satisfaisante pour le territoire de Saint-Martin, car elle ne prend pas en compte le degré d'endommagement des bâtiments. Or, les deux sources de données utilisées (SERTIT et COPERNICUS) permettant de connaitre l'impact d'Irma sur les bâtiments précisent le degré 
d'endommagement. C'est pourquoi il semblait pertinent de compléter la méthode California grâce aux connaissances acquises sur le terrain. Dans la mesure où les déchets n'ont quasiment pas été triés et traités après l'ouragan Irma, et au vu des photos prises sur le terrain, on considère que, dans une maison détruite, les déchets sont composés du toit, des fenêtres et des meubles et matériels électroniques du dernier étage. Nous avons alors estimé que les tas produits recouvraient l'espace sur plus d'un mètre en moyenne. Pour les bâtiments fortement endommagés, cette hauteur semblait en revanche au moins deux fois moindre.

La méthode que nous proposons est alors la suivante :

Estimation du volume de déchets pour un Bâtiment détruit

\section{Volume de déchet $=$ Surface $*$ A0 $\quad A 0=1,15$}

Hypothèse : $1,15 \mathrm{~m}$ de déchet si détruit

Estimation du volume de déchets pour un Bâtiment fortement endommagé

Volume de déchet $=$ Surface $*$ BO $\quad$ BO $=0,5$

Hypothèse : 0,5m de déchet si fortement endommagé

Les valeurs retenues pour A0 et B0 constituent des hypothèses de travail qu'il conviendra bien entendu de confirmer lors d'évènements semblables. Ces hypothèses pourraient également différer en fonction des territoires étudiés et des types de construction. En partant de ces valeurs et après un travail d'appariement des données du SERTIT avec les données des bâtiments de la BDTopo de l'IGN, nous avons comparé les volumes calculés par la méthode proposée avec ceux provenant des témoignages recueillis dans la presse et auprès de la décharge (Tableau 3). La méthode développée pour estimer les volumes de déchets générés par Irma à partir de la connaissance des bâtiments détruits (appelée «méthode DéPOs »), malgré son caractère très sommaire, permet de retrouver en partie les estimations provenant du terrain.

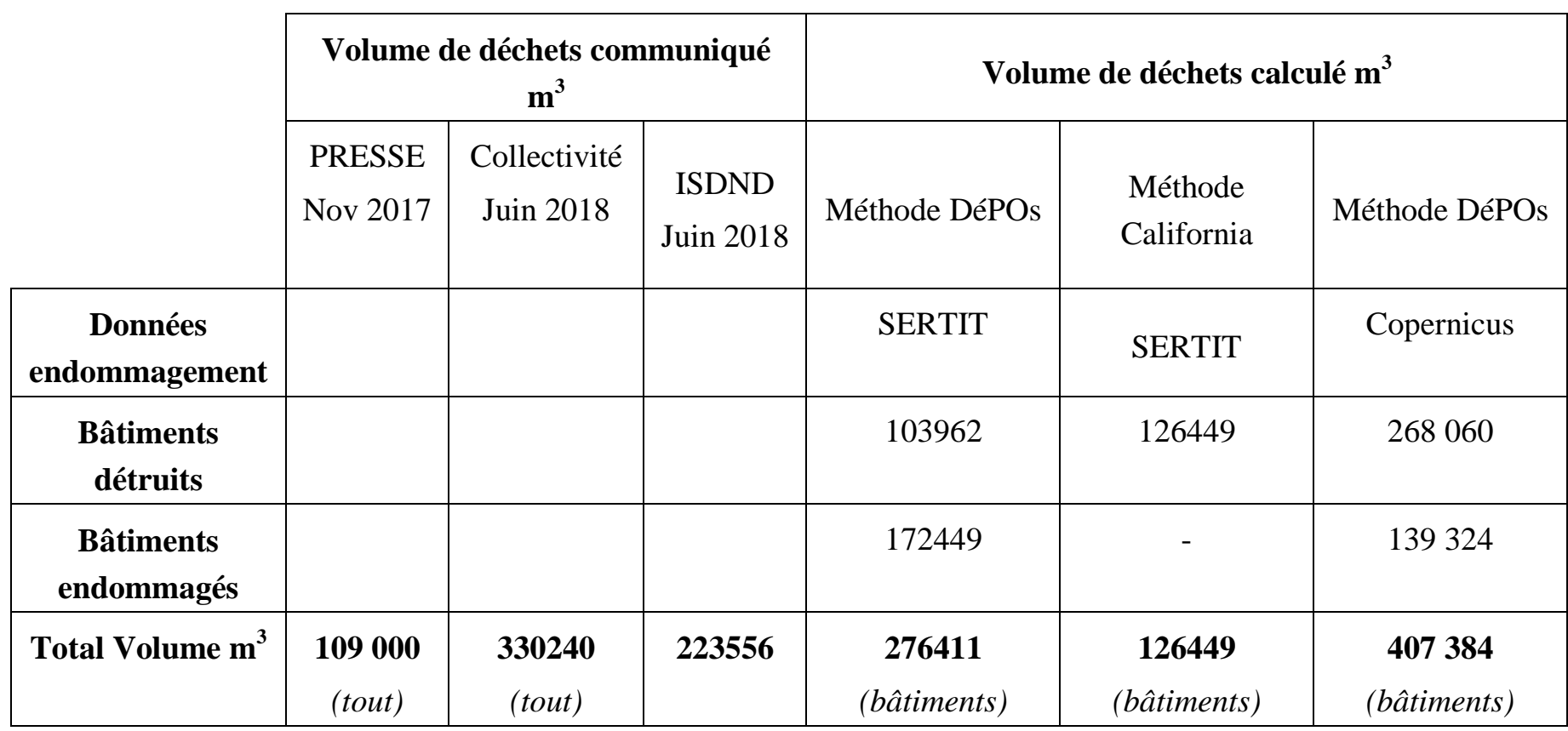

Tableau 3. Estimation des volumes de déchets générés par l'ouragan Irma à Saint-Martin selon les sources, dates et types.

La différence en volume de déchets entre les sources SERTIT $\left(\sim 280000 \mathrm{~m}^{3}\right)$ et COPERNICUS $\left(\sim 405000 \mathrm{~m}^{3}\right)$ est tout de même importante. Une analyse des photos montre qu'effectivement, COPERNICUS a classé davantage d'objets en détruit que le SERTIT. C'est pourquoi, il semble que l'on peut conclure que le volume des déchets provenant des bâtiments et générés par Irma sur SaintMartin est compris entre 300000 et $400000 \mathrm{~m}^{3}$, ce qui est cohérent avec l'estimation de la 
collectivité en juin 2018 qui estime le volume de déchets de $330240 \mathrm{~m}^{3}$ en intégrant tout type de déchets. C'est aussi cohérent avec la déclaration de la décharge (ISDND) qui déclare avoir reçu 223 $556 \mathrm{~m}^{3}$ en juin 2018 ou une partie des déchets provenant d'Irma n'a pas encore été acheminée à la décharge puisque les maisons détruites mettent plus de temps à être reconstruites en raison des assurances et chantiers de reconstruction et donc que les déchets de ces maisons peuvent arriver dans le cycle de la collecte bien plus tard. Ainsi malgré les nombreuses imprécisions, on peut considérer que la méthode DéPOs donne un ordre de grandeur acceptable que l'on pourra utiliser pour nos simulations.

\section{Localisation des bâtiments détruits et fortement endommagés}

Des analyses ont été menées sur la localisation des bâtiments détruits et fortement endommagés par Irma à partir des données du SERTIT afin d'être en mesure de mieux appréhender la localisation des gisements de déchets. Il apparaît alors clairement que la répartition des bâtiments détruits et fortement endommagés suit la répartition globale des autres bâtiments (Figure 6). La densité des bâtiments semble ainsi être le premier critère de répartition spatiale des dégâts, les impacts se concentrant là où les enjeux se concentrent.

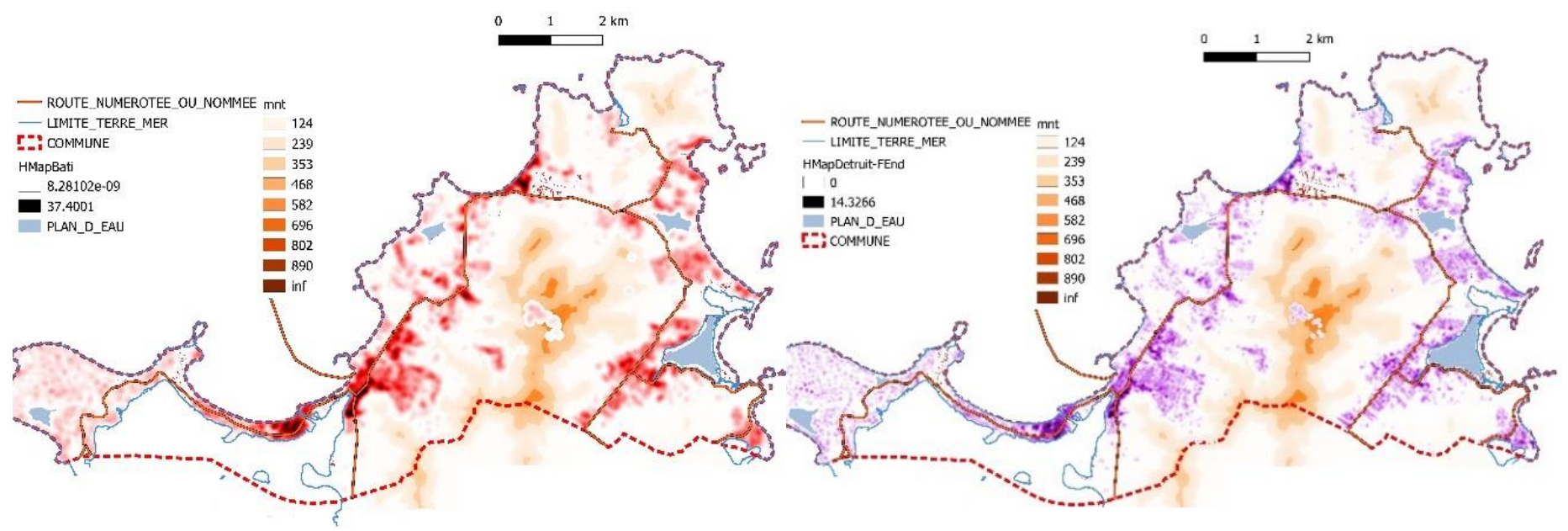

Figure 6. Densité des bâtiments (gauche) et densité des bâtiments détruits et fortement endommagés (droite).

Une analyse des pourcentages de bâtiments détruits et fortement endommagés a ensuite été réalisée en fonction de la distance à la mer. En effet, l'hypothèse d'un impact de la houle générée par Irma sur les bâtiments a très vite émergé pour tenter d'expliquer les impacts d'Irma dans certaines zones, les images impressionnantes d'impacts concernant majoritairement les zones côtières. L'analyse des données (tableau 4) semble montrer qu'il y a bien un effet «front de mer » qui disparaît néanmoins très rapidement quand on rentre un peu plus dans les terres. Il est en revanche difficile de conclure sur un effet dû au relief dans la mesure où il y a peu d'habitations dans le relief élevé de l'Ile, l'hypothèse sous-jacente étant que le relief contribue à protéger certaines zones des vents et bien entendu de la submersion. On peut aussi noter que les dégâts sont de tous côtés (à l'Est, au Nord et à l'Ouest), ce qui n'est pas étonnant puisque Saint-Martin s'est trouvé dans l'œil de l'ouragan. C'est donc bien l'ensemble de Saint-Martin qui a été touché. 


\begin{tabular}{|c|c|c|c|c|c|c|c|c|}
\hline \multirow{2}{*}{$\begin{array}{l}\text { Zone considérée } \\
\text { par rapport à la } \\
\text { distance à la mer } \\
\text { (m) }\end{array}$} & \multirow{2}{*}{$\begin{array}{c}\text { Nb de } \\
\text { bâtiments } \\
\text { dans cette } \\
\text { zone }\end{array}$} & \multirow{2}{*}{$\begin{array}{c}\text { \% des } \\
\text { bâtiments } \\
\text { de l'île } \\
\text { situés dans } \\
\text { cette zone }\end{array}$} & \multicolumn{3}{|c|}{ Bâtiments détruits } & \multicolumn{3}{|c|}{$\begin{array}{l}\text { Bâtiments fortement } \\
\text { endommagés }\end{array}$} \\
\hline & & & $\mathrm{nb}$ & $\begin{array}{l}\% \text { des } \\
\text { bati. de } \\
\text { la zone }\end{array}$ & $\begin{array}{c}\% \text { des } \\
\text { bati. } \\
\text { Détruits }\end{array}$ & $\mathrm{nb}$ & $\begin{array}{c}\% \text { bati. } \\
\text { de la } \\
\text { zone }\end{array}$ & $\begin{array}{l}\% \text { des } \\
\text { bati. } \\
\text { f-end }\end{array}$ \\
\hline $0-100$ & 1988 & 20.7 & 168 & 8,5 & 25,5 & 503 & 25,3 & 30,9 \\
\hline $0-200$ & 3596 & 37.5 & 255 & 7,1 & 38,7 & 787 & 21,8 & 48,5 \\
\hline $0-300$ & 4507 & 47 & 313 & 6.9 & 47,5 & 937 & 20,7 & 57,7 \\
\hline $0-500$ & 5970 & 62.3 & 424 & 7,1 & 64,3 & 1143 & 19,1 & 70,4 \\
\hline $0-1000$ & 8020 & 83.6 & 569 & 7,1 & 86,3 & 1449 & 18,1 & 89,2 \\
\hline Toute l'île & 9587 & 100 & \multicolumn{3}{|c|}{659} & \multicolumn{3}{|c|}{1624} \\
\hline
\end{tabular}

Tableau 4. Résumé statistique sur les tous les bâtiments. Source : Données Bâtis-SERTIT.

Enfin, si on opte pour une analyse par quartiers, le quartier le plus à l'ouest (Terres Basses) apparait visuellement moins touché que les autres (Figure 6 et 7). Or, ce territoire est réputé pour être un quartier aisé de l'île. Ainsi, il apparait rapidement que la composition des quartiers (aménagements, constructions, caractéristiques socio-économiques) peut être un facteur explicatif des impacts générés par Irma. Parmi les quartiers fortement touchés par l'ouragan, on identifie facilement les quartiers de Sandy Ground ou le Quartier d'Orléans, alors que le quartier de Terres Basses l'est effectivement sensiblement moins. Or, il y a bien entre ces quartiers de grandes différences socio-économiques que l'on voit déjà par la taille moyenne des habitations et par la densité (Tableau 5). Les quartiers de Sandy Ground et d'Orléans ont été désignés quartiers prioritaires de la politique de la ville par le décret 2014-1751 du 30 décembre 2014, alors que le quartier de Terres Basses est un quartier sans commerce, composé d'habitat dispersé dont des résidences en location haut de gamme.

\begin{tabular}{|l|c|c|c|c|c|c|c|c|}
\hline Nom & $\begin{array}{c}\text { Nombre de } \\
\text { bâtiments }\end{array}$ & $\begin{array}{c}\text { Surface } \\
\text { moyenne } \\
\text { bâtiments } \\
\mathrm{m}^{2}\end{array}$ & $\begin{array}{c}\text { E-type } \\
\text { surface } \\
\mathrm{m}^{2}\end{array}$ & $\begin{array}{c}\text { Densité du } \\
\text { bâti } \\
\text { (surf-bâti / } \\
\text { surface) }\end{array}$ & $\begin{array}{c}\text { Nombre } \\
\text { bâtiments } \\
\text { détruits }\end{array}$ & $\begin{array}{c}\text { \% } \\
\text { bâtiments } \\
\text { détruits }\end{array}$ & $\begin{array}{c}\text { Nombre } \\
\text { bâtiments } \\
\text { fortement } \\
\text { endommag. }\end{array}$ & $\begin{array}{c}\text { \% bati } \\
\text { fortement } \\
\text { endommag. }\end{array}$ \\
\hline $\begin{array}{l}\text { Sandy } \\
\text { Ground }\end{array}$ & 1756 & $\mathbf{1 1 1}$ & 166 & $\mathbf{1 9 . 4}$ & 80 & 4,6 & 235 & $\mathbf{1 3 . 4}$ \\
\hline $\begin{array}{l}\text { Terres } \\
\text { Basses }\end{array}$ & 1163 & $\mathbf{1 3 6}$ & 154 & $\mathbf{4 . 3}$ & 37 & 3,2 & 80 & 6.9 \\
\hline Orléans & 2736 & $\mathbf{8 5}$ & 104 & 6.8 & 168 & $\mathbf{6 . 1}$ & 362 & $\mathbf{1 3 . 2}$ \\
\hline St Martin & 17063 & 115 & 186 & 4,2 & 659 & 3.9 & 1624 & 9.5 \\
\hline
\end{tabular}

Tableau 5. Comparaison quartiers de Sandy Ground, de Terres Basses et d'Orléans.

Cette hypothèse reste néanmoins à vérifier, car il apparaît que le quartier de 'Terres Basses' est en fait le quartier le plus élevé de tous les quartiers habités de l'île, alors que les habitations de Sandy Ground sont situées juste à 1 ou 2 mètres au-dessus du niveau de la mer (figure 7). On peut 
d'ailleurs vérifier grâce à une carte du CEREMA (figure 8) que les quartiers de Sandy Ground et d'Orléans sont en zones inondables en cas de submersion marine, ce qui n'est pas le cas du quartier de Terres Basses. Les quartiers de Sandy Ground et d'Orléans sont donc conjointement plus populaires et plus exposés aux submersions que celui de Terres Basses. Existe-t-il un lien entre ces deux caractéristiques ? Cela pourra être analysé dans de futures recherches, à l'heure actuelle nous ne pouvons conclure.

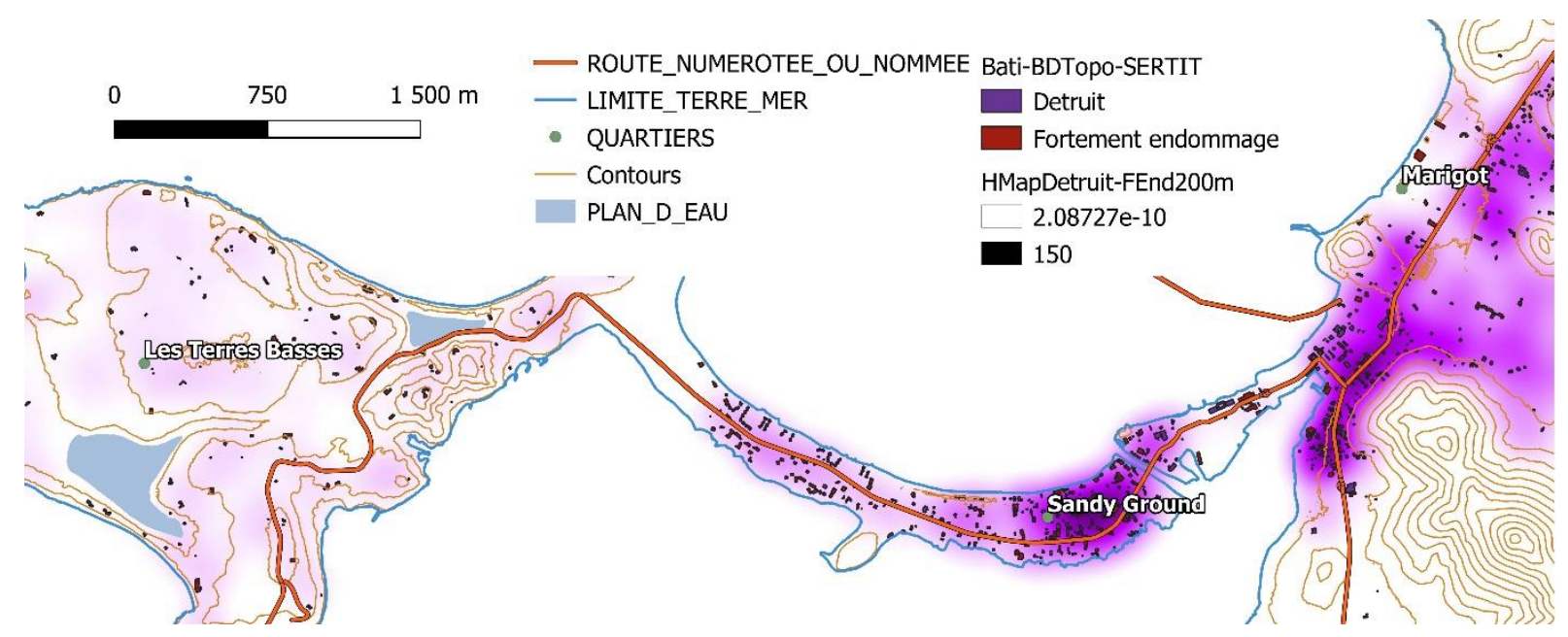

Figure 7. Courbes de niveaux (20m) sur les quartiers de Terres Basses, Sandy Ground et Marigot et la densité des maisons détruites et fortement endommagés.
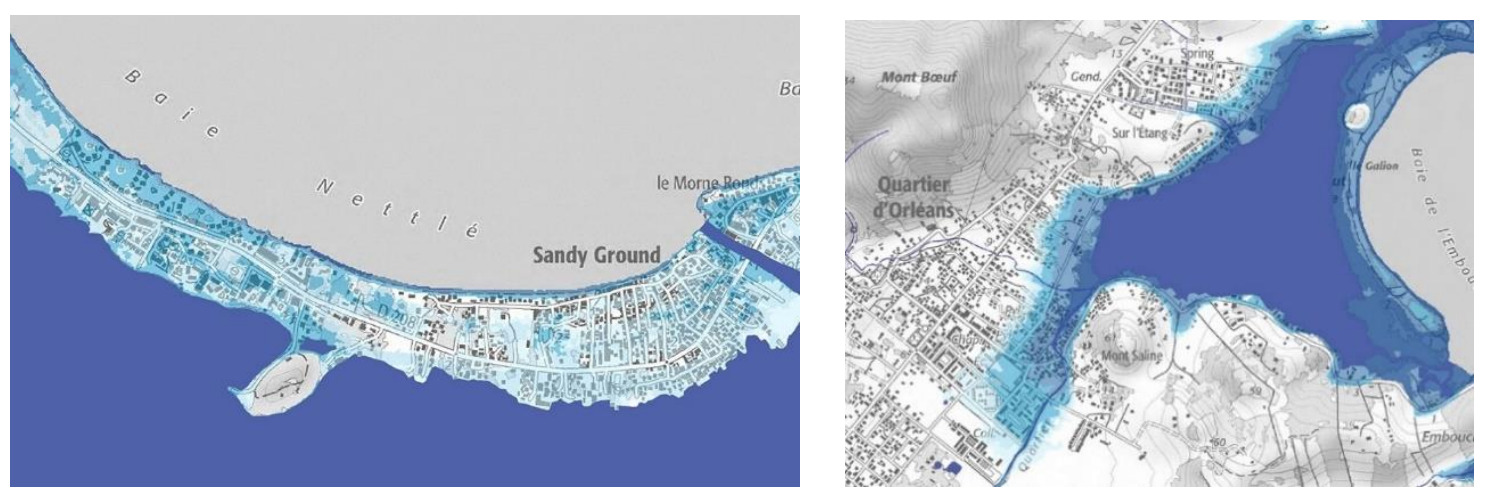

Figure 8. Extrait de la Carte des hauteurs de submersions marines sur Saint Martin suite à l'ouragan Irma. Les couleurs correspondent à des submersions de 2 et 3 m. (C) CEREMA, 21/11/2017

Enfin, une dernière question subsiste au regard de ces résultats: les habitations du quartier favorisé de Terres Basses ont-elles été plus épargnées en raison de leur positionnement géographique un peu en hauteur, loin des zones de submersions marines, ou sont-elles plus solides et plus résistantes aux ouragans ? Des recherches seraient à poursuivre pour étudier l'impact des constructions sur la vulnérabilité des habitations aux ouragans, ce qui contribuera à aider à développer des indicateurs de production des déchets post-ouragans.

\section{Perspectives}

Les travaux présentés dans cet article nous ont permis de concevoir un modèle d'analyse de la collecte des déchets post-ouragans ainsi qu'une fonction d'estimation des volumes de déchets lorsque les degrés d'endommagement des bâtiments sont connus. Nous avons également pu montrer que dans le cas d'Irma, les endommagements sont très corrélés à la densité des zones d'habitations, même si un effet de front de mer existe. On note toutefois que les quartiers populaires ont été les 
plus touchés ce qui s'explique très probablement par le fait qu'ils ont été construits sur des zones plus vulnérables.

D'autres recherches sont menées pour développer des méthodes d'estimation plus précises et plus robustes permettant de distinguer différents types de déchets pour le calcul des volumes de déchets (Marchesini et al., 2021). Par ailleurs, à partir de la localisation des bâtiments détruits et fortement endommagés et de la localisation des zones de transit et de la décharge (ISDND), nous avons conçu un algorithme de simulation de collecte de déchets qui sera présenté dans un article ultérieur. Cet algorithme nous permet de rejouer la collecte des déchets post Irma sur Saint-Martin et d'analyser ce qui pourrait permettre d'optimiser cette collecte pour de futurs ouragans, dont le nombre et la localisation des zones de transit ou la réduction des temps de chargement et déchargement. A termes ces recherches devront aboutir à la création d'un outil d'aide à la décision contribuant à mieux organiser la collecte des déchets post ouragans.

\section{Remerciement}

Les auteurs remercient l'Agence Nationale de la Recherche qui soutient le projet de recherche Déchets Post Ouragan (ANR-18-OURA-0003) et l'ensemble des partenaires de l'ANR DéPOs : Université Gustave Eiffel, Le Mans Université, Avignon Université, Association Robin des Bois et Université de Mons (partenaire international associé).

\section{Bibliographie}

Béraud H., 2013, Initier la résilience du service de gestion des déchets aux catastrophes naturelles: le cas des territoires urbains et de l'inondation. Thèse Université Paris-Est.

Beraud $\mathrm{H}$, Charlotte Nithart, Mathieu Durand, 2019 , Le difficile suivi des déchets post-catastrophe : le cas de l'Ouragan Irma à Saint-Martin, Risques urbains, ISTE Ltd., p24

Brown C, Milke M, \& Seville E. ,2011. Disaster management: A review article. Waste Management, vol. 31, Iss. 6 , 1085-1098.

Copernicus, EMNS 049, 2018, Reconstruction monitoring of St Martin and St Barthélemy islands (post IRMA) Stage01-Intermédiate report Accessibles sur le site https://emergency.copernicus.eu/ mapping/list-ofcomponents/EMSN049

Cangialosi John P., Latto Andrew S., and Berg Robbie, 2018, Hurricane Irma, National Hurricane Center 30 June 2018 https://www.nhc.noaa.gov/data/tcr/AL112017_Irma.pdf

Chen J.R., Tsai H.Y., Hsu P.C., Shen C.C., Estimation of waste generation from floods, Waste Management, Vol. 27, pp. 1717-1724.

Federal Emergency Management Adgency (FEMA), 2018, Hazus Earthquake Model User Guidance.

Gustin P, 2017, Repenser les Iles du Nord pour une reconstruction durable, rapport interministeriel. Rapport accessible sur le site http://www.outre-mer.gouv.fr/sites/default/files/rapport_de_philippe _gustin_delegue_interministeriel_a_la_reconstruction_21_novembre_2017.pdf

Hirayama N., Shimaoka T., Fujiwara T., Okayama T., Kawata Y., 2010, Establishment of disaster debris management based on quantitative estimation using natural hazard maps, WIT Trans. Ecol. Environ., Vol. 140, pp. 167-178

Lauritzen E.K., 1998, Emergency construction waste management, Safety Science, 30, pp. 45-53

Marchesini G, Beraud H, Barroca B, 2021, Quantification of disaster waste: Review of the available methods.. International Journal of Disaster Risk Reduction, Elsevier, 2021, 53, 〈10.1016/j.ijdrr.2020.101996〉. 〈hal$03154258\rangle$

Mc Entire D.A., 2006, Managing debris successfully after disaster: considerations and recommendations for Emergency Managers, Journal of Emergency Management, Vol. 4, pp. 23-8.

Nithart C., J. Bonnemains, 2018, Les dechets de l'ouragan Irma, Rapport ANR projet DéPOs, Octobre 2018

Office of Emergency Service OES CALIFORNIA, 2005, Débris Management in Disaster Recovery, OES, California 
Perez J., 2019, Gestion des dechets post-ouragan Irma Saint-Martin : Jeux d'acteurs et transformation du système loca d'avant, durant et après crise, Rapport de Master 2019

Popescu R. et J. Perez, 2019, Rapport de mission n² du projet ANR DéPOs.

Tabata T., Onishi A., Saeki T., Tsai P., 2019, Earthquake disaster waste management reviews: prediction, treatment, recycling, and prevention, International Journal of Disaster Risk Reduction, 36, pp. 101-119

Vinet F., Peroche M., Palany P., Leone F., Gherardi M., Grancher D., Moatty A., Defossez S., Collecte et gestion des débris post-cycloniques à Saint-Martin (Antilles françaises) après le passage du cyclone Irma (sept. 2017), Cybergeo : European Journal of Geography.

USEPA, 2008. Planning for natural disaster debris. In: Office of Solid Waste and Emergency Response and Office of Solid Waste (Eds.)

Zhang F., Cao C., Li C., Liu Y., Huisingh D., A systematic review of recent developments in disaster waste management, Journal of Cleaner Production, 235, pp. 822-840. 